\title{
CROMOBLASTOMICOSE PRODUZIDA POR FONSECAEA PEDROSOI NO ESTADO DO MARANHÃO. I - ASPECTOS CLÍNICOS, EPIDEMIOLÓGICOS E EVOLUTIVOS
}

\author{
Ana Carla C. Mello e Silva, Artur Serra Neto, Cloves Eduardo S. Galvão, \\ Sirley G. Marques, Ana Cristina R. Saldanha, Conceição de Maria Pedroso e \\ Silva, Olga Fischman, Raimunda Ribeiro da Silva, Maria do Rosário da S. R. \\ Costa e Jackson Maurício Lopes Costa
}

\begin{abstract}
Com o objetivo de verificar o comportamento clínico-epidemiológico da cromoblastomicose no Estado do Maranhão, foi feito um estudo retrospectivo e prospectivo de 13 casos, no serviço de Doenças Infecciosas e Parasitárias do Hospital dos Servidores do Estado do Maranhão no período de nov/88 a julho/91. Para a investigação, foi utilizada uma ficha protocolo com todos os dados necessários para uma análise posterior. Nos casos analisados observou-se inaior prevalência na faixa etária entre 50 e 60 anos $(46,1 \%)$ e do sexo masculino $(84,6 \%)$. Doze pacientes eram procedentes do Estado do Maranhão, dentre os quais 10 da microrregião da baixada ocidental maranhense. Quanto à profissão, $12(92,3 \%)$ eram lavradores. Na sua maioria apresentavam as lesões nos membros inferiores de forma verrugoconfluentes, cor acastanhada, com prurido. O tempo de evolução variou de $O$ a 15 anos em 12 casos (92,3\%). Quanto aos aspectos laboratoriais, o exame histológico feito em 12 pacientes, diagnosticando cromoblastomicose em $100 \%$ deles e a cultura isolou Fonsecaea pedrosoi em 9 casos (70\%). O tratamento realizado em todos os pacientes, com algumas variaçôes foi feito com 5 - fluorocitosina apresentando bons resultados evolutivamente. Constatou-se neste trabalho uma provável zona endêmica de cromoblastomicose na microrregião da baixada ocidental maranhense, até agora desconhecida.
\end{abstract}

Palavras-chaves: Cromoblastomicose. Fonsecaea pedrosoi. Estado do Maranhão.

A cromoblastomicose constitui uma micose subcutânea de aspecto polimorfo, acometendo a pele, tecido celular subcutâneo e, menos frequentemente a mucosa. Os trabalhadores rurais do sexo masculino, são as grandes vítimas desta moléstia, a qual interfere de maneira considerável em sua atividade ocupacional e capacidade de produção $^{79}$.

A doença foi estudada por diversos autores, daí receber várias denominações, tais como: dermatite verrucosa cromomicótica, pé musgoso, formigueiro, susina, blastomicose negra, doença de Pedroso, doença de Carrion e muitos outros. Os fungos comprovados como agentes etiológicos

\footnotetext{
Trabalho realizado no Departamento de Patologia, Universidade Federal do Maranhão, Hospital dos Servidores do Estado do Maranhão, São Luís, MA e Escola Paulista de Medicina, São Paulo, SP.

Enderefo para correspondência: Prof. Jackson Maurício Lopes Costa, Depto. de Patologia/UFMA. P̧. Madre Deus 02 (Anexo Hospital Geral), 65025-560 São Luís, MA.

Recebido para publicação em 20/08/91.
}

desta micose incluem: Fonsecaea pedrosoi, Fonsecaea compacta, Rhinocladiella aquaspersa, Phialophora verrucosa, Cladosporium carrioni e Exophiala jeanselmei, apresentando predileção por regiões de clima tropical subtropical e úmido, sendo encontrados na natureza, isolados do solo, do ar e dos vegetais ${ }^{7} 1516$.

A cromoblastomicose distribui-se geograficamente pelas zonas rurais dos países tropicais e subtropicais, provavelmente pela maior exposição destas populações ao agente infectante, principalmente através de ferimentos penetrantes nos membros inferiores. A distribuição mundial ocorre sob a forma de casos esporádicos em várias regiões, como: América Central, Antilhas, Sul dos Estados Unidos, Ilhas do Sul do Pacífico, América do Sul, Moçambique, Austrália e África do Sul7 131523 .

O Brasil ocupa um lugar de destaque em relação à freqüência de casos de cromoblastomicose, ocorrendo de forma endêmica em várias regiões, 
Silva ACCM, Neto AS, Galvão CES, Marques SG, Saldanha ACRS, Silva CMP, Fïschman O, Silva RR, Costa MRSR, Costa JML. Cromoblastomicose produzida por Fonsecaea pedrosoi no Estado do Maranhão. I - Aspectos clínicos, epidemiológicos e evolutivos. Revista da Sociedade Brasileira de Medicina Tropical 25:37-44, jan-mar, 1992.

ressaltando os estados da região Amazônica, Rio Grande do Sul, São Paulo, Rio de Janeiro e Minas Gerais como áreas de maior concentração de $\operatorname{casos}^{7} 101416$.

Dos agentes micóticos responsáveis pela cromoblastomicose no Brasil, Fonsecaea pedrosoi destaca-se na literatura como o grande causador da doença em nosso país ${ }^{7911}$. No Maranhão, apesar de não haver registros de casos na literatura, observaseum númerocrescente de ocorrências, necessitando de inquérito mais aprofundado sobre a doença no Estado.

Portanto, partindo dessa realidade, pretende-se neste trabalho demonstrar o comportamento clínico, epidemiológico e evolutivo de 13 casos de cromoblastomicose, observados no serviço de doenças infecciosas e parasitárias do Hospital do Servidores do Estado do Maranhão, no período de novembro de 1988 a julho de 1991 .

\section{MATERIAL E MÉTODOS}

Em um intervalo de tempo de 32 meses, foram acompanhados evolutivamente (ambulatório e enfermaria), 13 pacientes com cromoblastomicose, no Hospital dos Servidores do Estado do Maranhão. Para uma melhor análise dos mesmos foi feito um levantamento dos prontuários desde a primeira consulta ou internação à última avaliação, (julho/91).

Os dados colhidos dos prontuários destacavam: dados pessoais (idade, sexo, raça; naturalidade, procedência, profissão), tempo de doença, dados referentes às lesões (localização, aspecto, cor) $\mathrm{e}$ evolução clínica no decorrer da doença, correlacionando-a com a terapêutica empregada.

Para o diagnóstico laboratorial, foi feito uma biópsia com retirada de um fragmento do bordo ativo de uma lesão de cada paciente, sendo a amostra dividida em 2 partes, e estas armazenadas em recipientes contendo respectivamente, formol a $10 \%$ para o exame histopatológico e soro fisiológico a $0,9 \%$ para o exame micológico direto e cultura.

Exame histopatológico: o material obtido da biópsia era submetido a vários cortes de 5 nicra de espessura, que-em seguida eram corados pela hematoxilina-eosina e quando necessário pelo Giemsa e PAS e levados para observação em microscópio óptico.

Exame micológico direto: um pequeno fragmento era tratado com $\mathrm{KOH}$ a $20 \%$ em lâmina para o exame ao microscópio óptico, observando-se a presença dos corpúsculos escleróticos, quando positivo.

Cultura: O semeio era feito em meio AgarSaboroud + Cloranfenicol ou meio microbiótic agar (micoseel), esperando-se em média 2 semanas para obter crescimento das colônias, caracterizadas por serem aveludadas, negras ou violáceas de contornos nítidos. Realizou-se, ainda, o microcultivo segundo Ridell e col ${ }^{18}$.

\section{RESULTADOS}

Observou-se que a faixa etária predominantemente acometida foi a situada entre os 50 - 60 anos $(46,1 \%$ dos casos), e o sexo masculino destacou-se em maior proporção. Dos 13 casos estudados, 12 eram naturais do Maranhão, sendo $10(76,9 \%)$ procedentes da mircorregião da baixada ocidental maranhense. Com relação à ocupação profissional, 12 (92,3\%) eram lavradores e todos apresentavam como hábito comum o contato com a terra e vegetais. A Tabela 1 traz detalhes com relação aos dados pessoais dos pacientes envolvidos no estudo.

Quanto ao tempo de doença, verificou-se que 12 pacientes $(92,3 \%)$ enquadravam-se entre $0-15$ anos de evolução. Os membros inferiores foram acometidos em sua grande maioria $(84,6 \%)$. No que diz respeito aos aspectos morfológicos das lesões, a forma verrucosa confluente, foi observada em $100 \%$ dos casos, assim como a cor acastanhada das lesões também foi verificada em todos. Os dados acima estão apresentados na Tabela 2.

No que se refere aos exames laboratoriais constatou-se que: nos exames histopatológicos realizados, houve $100 \%$ de positividade para cromoblastomicose, apresentando os seguintes aspectos microscópicos: epiderme com hiperceratose, hipergranulose, acantose, hiperplasia pseudo-epiteliomatosa e exocitose; derme com intenso infiltrado inflamatório constituído por linfócitos, plasmócitos e histiócitos ao lado de gigantócitos, multinucleares que fagocitam corpúsculos acastanhados, 
Silva ACCM, Neto AS, Galvão CES, Marques SG, Saldanha ACRS, Silva CMP, Fischman O, Silva RR, Costa MRSR, Costa JML. Cromoblastomicose produzida por Fonsecaea pedrosoi no Estado do Maranhão. I-Aspectos clínicos, epidemiológicos e evolutivos. Revista da Sociedade Brasileira de Medicina Tropical 25:37-44, jan-mar, 1992.

Tabela 1 - Características epidemiológicas dos 13 pacientes portadores de cromoblastomicose.

\begin{tabular}{lccllllll}
\hline Paciente & Idade & Sexo & Raça & Naturalidade & Procedência & Profissão & Hábitos \\
\hline FS & 59 & M & Branca & Maranhense & S. Bento (MA) & Lavrador & H1 H2 H3 \\
MCMA & 54 & F & Branca & Maranhense & S.V.Ferrer(MA) & Lavradora & H1 H2 \\
RIV & 48 & M & Parda & Cearense & S.Luís(MA) & Lavrador & H1 H2 H3 \\
GAM & 51 & M & Negra & Maranhense & Bacuri(MA) & Lavrador & H1 H2 \\
FNM & 39 & M & Parda & Maranhense & S.Luís(MA) & Carpinteiro & H1 & \\
JCB & 67 & M & Negra & Maranhense & Anajatuba(MA) & Lavrador & H1 H2 H3 \\
ACC & 51 & M & Parda & Maranhense & Guimarães(MA) & Lavrador & H1 H2 \\
HJR & 65 & M & Parda & Maranhense & Anajatuba(MA) & Lavrador & H1 H2 H3 \\
CSC & 57 & M & Parda & Maranhense & Cururupu(MA) & Lavrador & H1 H2 H3 \\
RNM & 37 & M & Parda & Maranhense & Itapecuru(MA) & Lavrador & H1 H2 H3 \\
AC & 55 & M & Parda & Maranhense & Anajatuba(MA) & Lavrador & H1 H2 H3 \\
FC & 51 & M & Branca & Maranhense & Sta.Helena(MA) & Lavrador & H1 H2 H3 \\
HGM & 39 & F & Parda & Maranhense & Bacuri(MA) & Lavradora & H1 H2 H3 \\
\hline
\end{tabular}

Chave de localização: Hábitos $\mathrm{H} 1$ - contato com a terra

$\mathrm{H} 2$ - andar descalço

H3 - microtraumatismo por funções habituais

arredondados, e refringentes com formação de microabscessos. O exame micológico direto feito em 12 dos pacientes, mostrou corpúsculos escleróticos ou fumagóides, redondos, de cor parda com paredes grossas e birrefringentes, isolados ou agrupados, em 10 casos. A cultura realizada com material obtido de 12 pacientes, apresentou crescimento de colônias enegrecidas ou oliváceas, aveludadas e planas com centro elevado, em 9 dos exames, tendo então a identificação de Fonsecaea pedrosoi e suas estruturas de frutificação (Cladosporim, Phialophora e Rhinocladiella) através do microcultivo.

O tratamento utilizado nos 13 pacientes foi a 5fluorocitosina na dose de 100 a $150 \mathrm{mg} / \mathrm{kg} / \mathrm{peso} / \mathrm{dia}$ divididos em 4 tomadas diárias por um período médio de 6 meses. A anfotericina B foi associada com a 5-fluorocitosina em 3 casos, não havendo resposta satisfatória, devido aos efeitos colaterais observados com esta associação.

Como terapêutica coadjuvante foi utilizada a exerese cirúrgica em 5 pacientes ( $38,4 \%$ dos casos), tendo em vista haver resposta insatisfatória ao tratamento clínico, asssim como nos pacientes portadores de lesões pequenas. Na avaliação final, julho de 1991, verificou-se os seguintes resultados: 1 paciente $(7,6 \%)$ com cura clínica (lesões totalmente cicatrizadas) e laboratorial (biópsia realizada após 6 meses da cicatrização com exame direto, cultura e histopatológico negativos); 11 pacientes $(84,6 \%)$ com quadro em involução e 1 paciente $(7,6 \%)$ sem controle. A Tabela 3 e as Figuras 1 e 2 trazem detalhes do tratamento realizado e 0 aspecto clínico da lesão cromomicótica em nosso Estado. 
Silva ACCM, Neto AS, Galvão CES, Marques SG, Saldanha ACRS, Silva CMP, Fischman O, Silva RR, Costa MRSR, Costa JML. Cromoblastomicose produzida por Fonsecaea pedrosoi no Estado do Maranhão. I-Aspectos clínicos, epidemiológicos e evolutivos. Revista da Sociedade Brasileira de Medicina Tropical 25:37-44, jan-mar, 1992.

Tabela 2 - Características clínicas das lesões observadas nos pacientes estudados.

\begin{tabular}{|c|c|c|c|c|c|c|c|}
\hline \multirow[t]{2}{*}{ Paciente } & \multicolumn{2}{|c|}{$\begin{array}{c}\text { Localização da lesão } \\
\text { Código I }\end{array}$} & \multirow[t]{2}{*}{$\begin{array}{l}\text { Tempo de doença } \\
\text { (anos) }\end{array}$} & \multicolumn{4}{|c|}{$\begin{array}{l}\text { Aspecto da lesão } \\
\text { Código II }\end{array}$} \\
\hline & 1 & 3 & & 1 & 2 & 3 & 4 \\
\hline
\end{tabular}

\begin{tabular}{|c|c|c|c|c|c|c|}
\hline FS & 1 & 5 & 1 & 2 & 3 & 4 \\
\hline MCMA & 2 & 15 & 1 & 2 & 3 & 4 \\
\hline GAM & 2 & 4 & 1 & 2 & 3 & 4 \\
\hline RJV & 2 & 14 & & & 3 & \\
\hline FMN & 2 & 1 & 1 & 2 & 3 & \\
\hline $\mathrm{JCB}$ & 2 & 3 & 1 & 2 & 3 & 4 \\
\hline HJR & 2 & 20 & & & 3 & 4 \\
\hline $\mathrm{ACC}$ & 2 & 3 & 1 & 2 & 3 & $*$ \\
\hline CSC & 3 & 10 & 1 & 2 & 3 & \\
\hline RNM & 2 & 10 & 1 & 2 & 3 & 4 \\
\hline $\mathrm{AC}$ & 2 & 4 & 1 & 2 & 3 & \\
\hline FC & 2 & 15 & & & 3 & 4 \\
\hline HGM & 2 & 3 & & & 3 & \\
\hline
\end{tabular}

Observação: todos os pacientes apresentaram lesões de cor acastanhada e não encontramos linfadenopatia associada.

Chave localização: (Código I) 1 - MMSS (membros superiores)

2 - MMII (membors inferiores)

3 - glúteo
(Código II) 1 - pápula
2 - verrucosa nodosa
3 - verrucosa confluente
4 - verrucosa ulcerada

\section{DISCUSSÃO}

Por ser considerada uma doença crônica pelos autores, com um tempo de evolução das lesões, em média, entre 10 e 20 anos, é de se esperar que os pacientes acometidos pela cromomicose sejam adultos, com idade variável entre 30 e 50 anos, embora ocorressem variações como as observadas neste trabalho, em que $75 \%$ dos pacientes tinham idade superior a 50 anos e tempo de doença entre 
Silva ACCM, Neto AS, Galvão CES, Marques SG, Saldanha ACRS, Silva CMP, Fischman O, Silva RR, Costa MRSR, Costa JML. Cromoblastomicose produzida por Fonsecaea pedrosoi no Estado do Maranhão. I - Aspectos clínicos, epidemiológicos e evolutivos. Revista da Sociedade Brasileira de Medicina Tropical 25:37-44, jan-mar, 1992.

Tabela 3 - Distribuição quanto ao tempo de doença dos 13 pacientes estudados correlacionados com o tratamento e evolução.

Total $\begin{gathered}\text { Número de } \\ \text { pacientes }\end{gathered} \quad \begin{gathered}\text { Tempo de } \\ \text { doença }\end{gathered} \quad$ Tratamento

involução cura s/controle

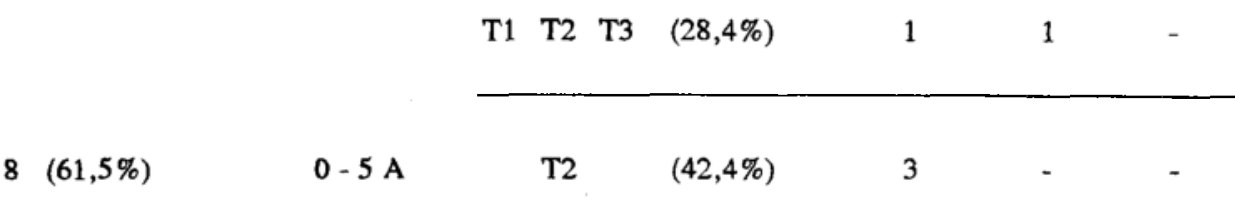

13

T2 T3 $(14,2 \%) \quad 1$

$100 \%$

T1 T2 $(14,25 \%)$

2

T1 T2 T3 $(20,0 \%) \quad 1$

$\begin{array}{llllll}4(30,7 \%) & 6-15 \mathrm{~A} & \mathrm{~T} 2 & (80,0 \%) & 2 & -\end{array}$

$1(7,6 \%) \quad 16-20 \mathrm{~A} \quad \mathrm{~T} 2 \quad(100,0 \%)$

Chave localização: Tratamento T1 - exérese cirúrgica

T2 - 5-Fluorocitosina

T3 - anfotericina B

0 e $5 \operatorname{anos}^{7} 1220$.

A maioria dos casos ocorre em países de clima tropical e subtropical, predominando no Brasil ${ }^{9} 10$ 1213 . Os trabalhadores rurais são as grandes vítimas da doença, pois, tomando como exemplo os nossos casos, temos que $92,3 \%$ dos pacientes tinham como atividade principal a lavoura, sendo o sexo masculino predominante comparado ao sexo feminino, concordando com $\mathrm{Lacaz}^{7}$, quando este refere que os trabalhadores rurais do sexo masculino pagam o maior tributo à infecção.

O tipo de atividade desempenha o fator preponderante na localização das lesões, pois observamos que em $84,6 \%$ dos nossos pacientes o comprometimento foi nos membros inferiores, havendo relatos de pequenos ferimentos por espinhos, madeiras, pedras, facilitando a penetração oportunística do fungo. Os nossos achados concordam com a maioria dos autores ${ }^{7} 101423$.

Segundo Tibiriçá ${ }^{21}$, os aspectos macroscópicos das lesốes podem ser classificados como: verrucosa nodosa (verrugas isoladas), verrucosa confluente e verrucosa ulcerosa ou úlceroverrucosa. Nos nossos casos pudemos notar o polimorfismo lesional com 
Silva ACCM, Neto AS, Galvão CES, Marques SG, Saldanha ACRS, Silva CMP, Fischman O, Silva RR, Costa MRSR, Costa JML. Cromoblastomicose produzida por Fonsecaea pedrosoi no Estado do Maranhão. I* Aspectos clínicos, epidemiológicos e evolutivos. Revista da Sociedade Brasileira de Medicina Tropical 25:37-44, jan-mar, 1992.

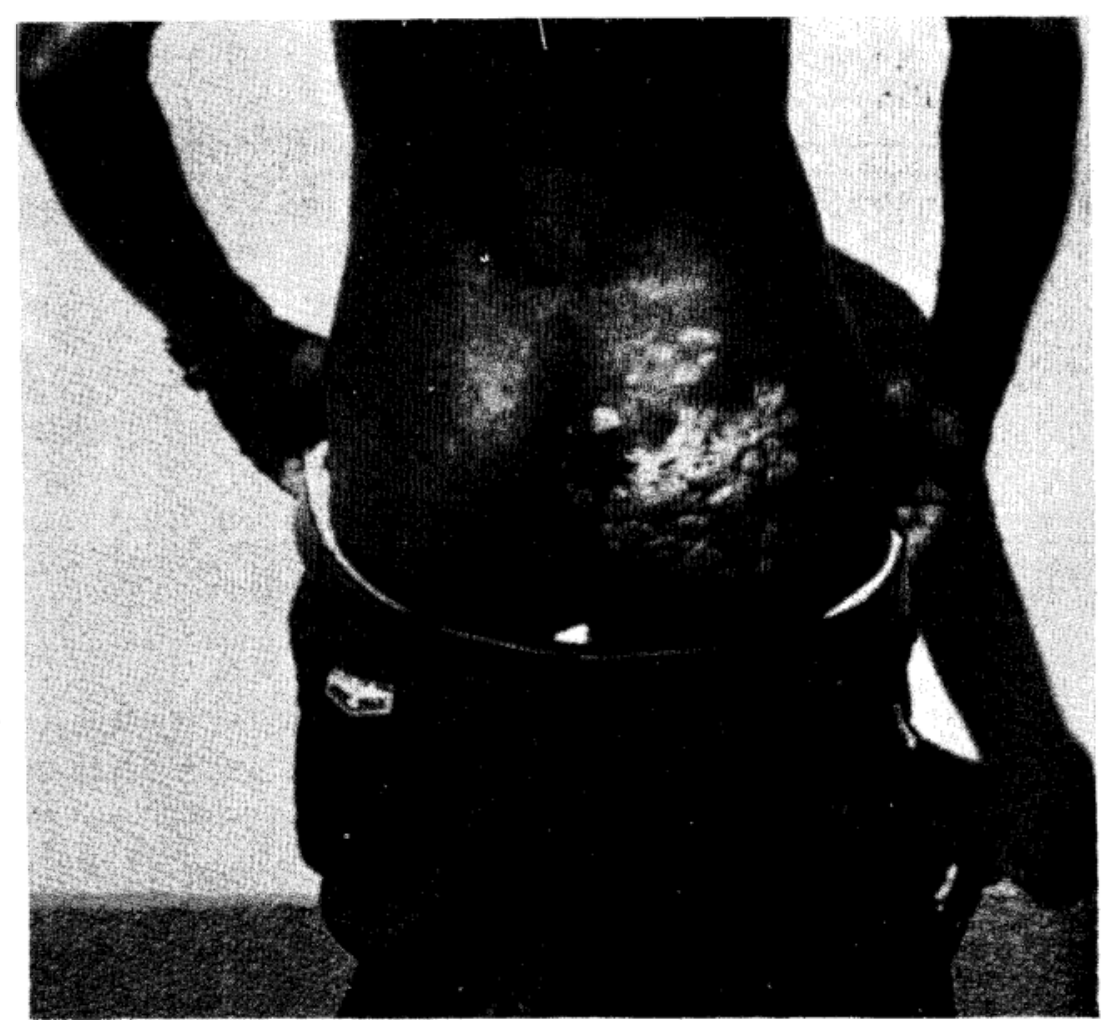

Figura 1 - Paciente CSC, apresentado lesões verrugo confluente ao nivel da região glútea esquerda em forma de placa gigante. Esta localização é pouco freqüente na cromoblastomicose.

predominância da forma verrucosa confluente. Chamou-nos a atenção a inexistência de adenopatias, o que concorda com a maioria dos autores ${ }^{8} 14$, embora Romero e Trejos ${ }^{19}$ na Costa Rica, Fadder e McGinnis $^{6}$ nos Estados Unidos e Silva ${ }^{20}$ no Brasil tenham encontrado índices variáveis de adenopatias em seus pacientes.

Outro dado importante que merece atenção é que dos 13 pacientes estudados, somente $3(23 \%)$ desenvolveram edema, e destes, apenas 2 eram do tipo elefantiásico, justamente os pacientes que desenvolveram infecção secundária durante o curso de sua doença. Na tentativa de explicar a elefantíase de alguns caso, Silva ${ }^{20}$ e Esteves cols ${ }^{5}$, referem a existência de um bloqueio linfático e de intensa proliferação fibrosa durante o curso da doença nestes pacientes.

A presença de 10 pacientes $(76,9 \%$ da causuística) procedentes da microrregião da baixada ocidental maranhense, zona reconhecidamente endêmica de esquistossomose mansônica ${ }^{4}{ }^{17}$, faz- nos pensar na existência de uma zona endêmica da doença em nosso Estado, a qual será estudada posteriormente em outro relato. Quanto à natureza dos agentes etiológicos encontrados, há preponderância do Fonsecaea pedrosoi, confirmando ser o fungo responsável pela maioria dos casos da doença no Brasil ${ }^{7} 1015$.

A abordagem terapêutica em doenças consideradas crônicas é sempre difícil para o clínico, pois, geralmente há necessidade de interações de drogas ou procedimentos ${ }^{123}$. Utilizamos em nossos pacientes, como medicação base, a 5-fluorocitosina, que é considerada a droga de primeira escolha no tratamento da cromoblastomicose por Fonsecaea pedrosoi. Droga utilizada em primeira instância por Lopes e cols em 1969, quando obtiveram 2 casos de cura $^{10}$. Também foram Lopes e cols os primeiros a registrarem o fenômeno de resistência à droga in vivo e in vitro de amostras isoladas de cinco doentes ${ }^{1012}$.

Observou-se que por ser um tratamento 
Silva ACCM, Neto AS, Galvão CES, Marques SG, Saldonha ACRS, Silva CMP, Fïschman O, Silva RR, Costa MRSR, Costa JML. Cromoblastomicose produzida por Fonsecaea pedrosoi no Estado do Maranhão. I - Aspectos clínicos, epidemiológicos e evolutivos. Revista da Sociedade Brasileira de Medicina Tropical 25:37-44, jan-mar, 1992.

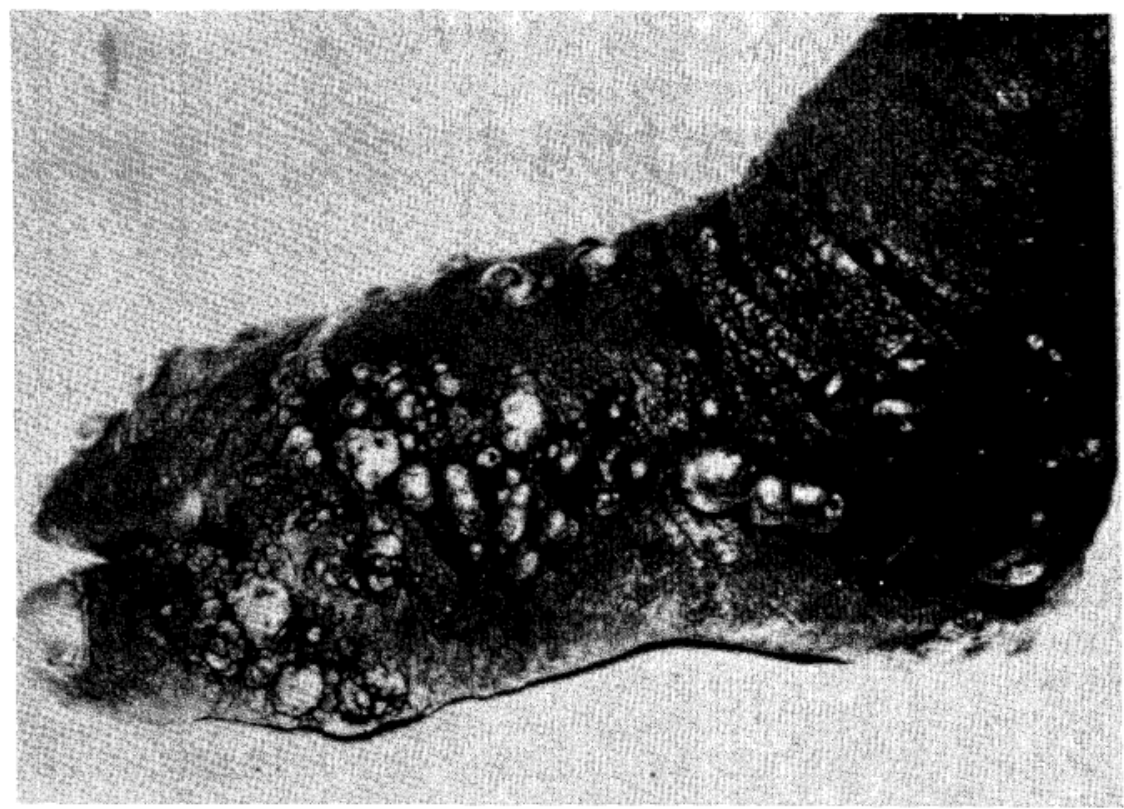

Figura 2 - Paciente ACC, apresentando edema no membro inferior com polimorfismo lesional, caracterizando-se por papulas, verrugas nodulares e verrugas confluentes.

prolongado e caro para os pacientes, pois a grande maioria é constituída de lavradores sem recursos, embora todo o tratamento tenha sido custeado pelo Hospital dos Servidores, tornou-se difícil o acompanhamento evolutivo dos pacientes, fato comum entre os autores 181122 .

A associação 5-fluorocitosina com anfotericina $B$ não se mostrou eficaz, tendo em vista os efeitos colaterais apresentados pelos pacientes. Testes in vitro realizados mostraram que anfotericina $B$ é inócua para os fungos responsáveis pelas lesões da cromoblastomicose ${ }^{14}$. Quanto à exerese cirúrgica associada a 5-fluorocitosina, observou-se bons resultados, principalmente em lesões únicas e de pequeno diâmetro.

\section{SUMMARY}

The aim of this study was to obse"ve the clinical and of epidemiological behavior of chromoblastomycosis in the State of Maranhão. A retrospective and prospective study of 13 cases was performed at the infectious diseases section of the Hospital dos Servidores do Estado do Maranhão, from November, 1988 to July, 1991. In the investigation a protocol record was used with a view to further analysis. A higher prevalence betweem 50 and 60 years of age $(46.1 \%)$ and in male patients $(92.3 \%)$ was noted. Twelve patients (92.3\%) were from Maranhão State, and 10 of them (76.9\%) came from the Western Microregion of the State of Maranhão. Regarding occupation, $92.3 \%$ were landworkers, and most of them presented the lesions on the lower limbs (84.6\%) of a vegetant warty aspect, brown in color with itching.

Histopathological exammination diagnosed chromomycosis in $100 \%$ of the cases. Culture was performed in 11 cases, and Fonsecaea pedrosoi isolated in 9 of them. Treatment with 5 -fluorocytosine resulted in a good evolutive response. This study indicates the existence of a probable endemic area of chromomycosis in hinterland of Maranhão (Western Microregion) that hither to unknown.

Key-words: Chromoblastomycosis. Fonsecaea pedrosoi. State of Maranhão.

\section{REFERÊNCIAS BIBLIOGRÁFICAS}

1. Borelli D. A clinical trial of itraconazole in the treatment of deep mycoses and leishmaniasis.
Reviews of Infectious Diseases 9:57-63, 1987.

2. Costa JML, Sampaio RN, Tada MS, Almeida EA, 
Silva ACCM, Neto AS, Galvão CES, Marques SG, Saldanha ACRS, Silva CMP, Fischman O, Silva RR, Costa MRSR, Costa JML. Cromoblastomicose produzida por Fonsecaea pedrosoi no Estado do Maranhão. I - Aspectos clínicos, epidemiológicos e evolutivos. Revista da Sociedade Brasileira de Medicina Tropical 25:37-44, jan-mar, 1992.

Veiga EP, Magalhães AV, Marsden PD. Furazolidone treatment of cutaneous leishmaniasis. Transactions of the Royal Society of Tropical Medicine and Hygiene 79:274, 1985.

3. Costa JML. Estudo clínico epidemiológico de um surto epidêmico de leishmaniose tegumentar americana na região de Corte de Pedra, ValençaBA. Tese de Mestrado, Universidade de Brasília, 1986.

4. Cutrim RNM. Aspectos clínico-epidemiológicos da esquistossomose mansoni em três localidades da baixada Ocidental Maranhense. Tese de Mestrado, Instituto Oswaldo Cruz, 1987.

5. Esteves JA, Cabrita JD, Nobre GN. Micologia médica. Fundação Colouste Gulbenkian, 1980.

6. Fader RC, McGinnis MR. Infections caused by dematiaceus fungi: Chromblastomycosis and phaeohyphomycosis infectious disease. Clinics of North America 2, 1988.

7. Lacaz CS, Porto E, Martins JEC. Micologia médica. Fundos, actinomicetos e algas de interesse médico. Editora Sarvier, São Paulo, 1984.

8. Lane CG. A cutaneous lesion caused by a new fungos (Phialophora verrucosa). Journal Cutaneous Disease 33:840-846, 1915.

9. Londero AT, Ramos CD. Chromomycosis: a clinical and mycologic study of thirty-five cases observed in the hinterland of Rio Grande do Sul, Brazil. The American Journal of Tropical Medicine and Hygiene 25:132-135, 1976.

10. Lopes CF, Alvarenga RJ, Cisalpino EO, Martinelli B, Santos PU, Armond $S$. Tratamento da cromomicose pela 5-flurocitosina. Primeiros resultados. O Hospital 75:1335-1342, 1969.

11. Lopes CF. Tratamento e cura da cromomicose pela 5-fluorocitosina. Anais Brasileiros de Dermatologia 48:165-171, 1973.

12. Lopes CF, Alvarenga RJ, Cisalpino EO, Resende MA, Oliveira LG. Six years experience in treatment of Chromomycosis with 5-fluorocytosine. Internal Journal Dermatology 17:414-418, 1978.

13. Lopes CF. Recent developments in the therapy of chromoblastomycosis. Bulletin of Panamerican Health Organization 15:58-64, 1981.

14. Mattêde MGS, Palhano Junior L, Coelho CC, Mattêd AF. Dermatite verrucosa cromoparasitária (cromomicose). Investigação de casos no Estado do Espírito Santo. Anais Brasileiros de Dermatologia 65:70-74, 1990.

15. McGinnis MR, Hilger EA. Infections Caused by black fungi. Archives Dermatology 123:1300-1302, 1987.

16. Pereira Filho MJ. O pé musgoso de Thomas e a cromoblastomicose no Rio Grande do Sul eem Santa Catarina; Identificação dos fungos patogênicos no gênero Phialophora, Thaxter, 1915. Resultados terapêuticos. Revista do Rio Grande do Sul 32:66101, 1949.

17. Pellon AB, Teixeira I. Distribuição da esquistossomose no Brasil. Divisão de Organização Sanitária, 1950.

18. Ridell GS, Laciow DM. Mycology for the clinical Laboratory. Reston. Reston Publications, 1979.

19. Romero A, Trejos A. La cromoblastomicosis en Costa Rica. Revista Biologia Tropical 1:95-115, 1953.

20. Silva NN. Cromoblastomicose no Rio Grande do Sul. Aspectos clínicos, micológico e experimental. Anais Brasileiros Dermatologia Sifilografia 24:113145, 1949.

21. Tibiriça POT. Anatomia patológica da dermatite verrucosa cromomicótica. Tese, Universidade de São Paulo, 1939.

22. Trejos A. Cladosporium carrionii n. sp. and the problem of Cladosporia isolated from chromoblastomycosis. Revista Biologia Tropical 2:75-112, 1954.

23. Vollum DI. Chromomycosis: a review. British Journal of Dermatology 96:454-458, 1977. 